\title{
Role of Inflammatory Cytokines in Insulin Resistance Among Chronic Hepatitis C Patients
}

\author{
Nouman Mohammed Elgarem ${ }^{1}$, Mohammad Ahmad Elghobary ${ }^{1}$, Rasha Hamed EI Sherif ${ }^{2}$, \\ Maha Assem Hussien ${ }^{1}$, Yousra Hamed Mourad ${ }^{1}$ \\ ${ }^{1}$ Departments of Internal Medicine, Faculty of Medicine, Cairo University, Cairo, Egypt \\ ${ }^{2}$ Clinical and Chemical Pathology, Faculty of Medicine, Cairo University, Cairo, Egypt
}

\section{Email address:}

Ngarem2@gmail.com (N. M. Elgarem),melghobary@yahoo.com (M. A. Elghobary), maha.assem@hotmail.com (M. A. Hussien), rasha.elsherif@Kasralainy.edu.eg (R. H. E. Sherif),dr.yousra_hamed@yahoo.com(Y.H. Mourad)

To cite this article:

Nouman Mohammed Elgarem, Mohammad Ahmad Elghobary, Rasha Hamed El Sherif, Maha Assem Hussien. Yousra Hamed Mourad. Role of Inflammatory Cytokines in Insulin Resistance Among Chronic Hepatitis C Patients. American Journal of Internal Medicine.

Vol. 4, No. 5, 2016, pp. 79-84. doi: 10.11648/j.ajim.20160405.11

Received: July 18, 2016; Accepted: August 2, 2016; Published: August 31, 2016

\begin{abstract}
Insulin resistance plays an essential role in the pathogenesis of diabetes associated with HCV. High levels of inflammatory cytokines have been found in $\mathrm{HCV}$-infected patients. The aim of the study was to investigate the association of $\mathrm{HCV}$ infection with impaired glucose metabolism and to highlight the role of inflammatory cytokines as an initial mechanism involved in insulin resistance development in HCV infection. It included 3 groups of patients: Group I: $50 \mathrm{HCV}$ patients with DM. Group II: $50 \mathrm{HCV}$ patients without DM and Group III: 25 patients with DM alone as control subjects. Insulin resistance was evaluated using the (HOMA- IR) index. We measured the levels of fasting insulin, CRP and two of the inflammatory cytokines of the innate immunity (TNF- $\alpha$ and IL-6 by ELISA). It was found that insulin resistance, CRP and IL6 in group (I) were significantly higher when compared to group (II) and (III) with P-value $<0.001$. CRP in group (II) was significantly higher when compared to group (III) with P-value $<0.05$. Serum level of (TNF- $\alpha$ ) in group (I) was significantly higher when compared to group (II) with P-value $<0.05$ and group (III) with P-value $<0.001$ as well as in group (II) it was significantly higher when compared to group (III) with P-value $<0.001$. CRP, IL-6 and TNF- $\alpha$ had statistically significant positive direct correlation to insulin resistance. In conclusion there was a strong relationship between inflammatory cytokines and the occurrence of insulin resistance in chronic HCV patients with type 2 diabetes mellitus.
\end{abstract}

Keywords: Diabetes, Inflammatory Cytokines, Insulin Resistance and Chronic HCV Infection

\section{Introduction}

"Diabetes and hepatitis C virus (HCV) infection are severe health problems in the developing countries. [1] Many studies have suggested that the prevalence of diabetes mellitus is high among patients with chronic hepatitis $\mathrm{C}$ with or without coexisting cirrhosis [2], [3] Insulin resistance which is induced by HCV seems to play a vital role in the pathogenesis of HCV- induced glucose intolerance [1]."

"Chronic inflammation plays a role in the development of insulin resistance through enhancing inflammatory cytokines. $\mathrm{HCV}$ establishes chronic infection through cellular targets involved in innate immunity [4] Cytokines of the innate immune response mainly IL-6 and tumor necrosis factor
(TNF)- $\alpha$ stimulate production of acute-phase proteins by the liver [3] In type 2 diabetes, there is an ongoing cytokinemediated acute-phase response and this is closely involved in the pathogenesis of the disease [4]."

The aim of our study was to investigate the association of $\mathrm{HCV}$ infection with impaired glucose metabolism and to highlight the role of inflammatory cytokines as an initial mechanism involved in insulin resistance development in $\mathrm{HCV}$ infection.

\section{Materials and Methods}

\subsection{Patients}

The study included 100 patients with chronic liver disease 
with Grade A score according to the modified Child-TurcottePugh score. They were recruited from the inpatient ward and the outpatient clinic of the Internal Medicine Hospital at Cairo University during the period from June 2013 to March 2014. The patients were divided into three groups. Group A included 50 chronic HCV patients with Type 2 DM, Group B included 50 chronic HCV patients without DM and Group C included 25 patients with type $2 \mathrm{DM}$ with matched; age, sex and disease duration as control subjects.

\subsection{Exclusion Criteria}

Patients with other concomitant hepatic viral infections (HBV), CLD stage B \& C according to modified ChildTurcotte-Pugh score, autoimmune hepatitis, hepatorenal syndrome, hepatic focal lesions, other coexisting medical illness and obese patients (BMI >30). All groups were carefully matched by age, sex, BMI, negative family history of type 2 diabetes, fasting blood glucose and severity of liver disease which was assessed according to Modified Child Pugh's classification.

\subsection{Ethical Aspects}

Research protocols were approved by the medical ethics committee of Kasralainy Medical School, Cairo University. All participants provided a written informed consent after the research protocols were explained to them. Informed consent was obtained from all the study participants and their approval was taken by signature.

\subsection{Procedures}

All participants underwent a complete screening panel, including medical history, clinical examination and measurement of body mass index.

Specimen collection: Blood samples were collected from each patient after 6-8 hours of overnight fasting using a sterile plastic syringe to withdraw venous blood by a single puncture technique of the most accessible vein. Samples were dispensed into 3 sterile tubes: The first tube contained K-EDTA solution $(1.2 \mathrm{mg} / \mathrm{ml})$ as an anticoagulant for complete blood picture. The second one contained sodium citrate solution $(3.2 \mathrm{mg} / \mathrm{ml})$ as an anticoagulant for prothrombin time assay. The third tube was without an anticoagulant (clotted sample) for biochemical investigations, serum TNF- $\alpha$ and IL- 6 . Serum was prepared by centrifugation for 10 minutes at $3000 \mathrm{rpm}$ at room temperature, aliquoted and stored after labeling at $-20^{\circ} \mathrm{C}$ until analysis.

Biochemical profile included the following: serum electrolytes ( $\mathrm{Na}, \mathrm{K})$, liver enzymes (ALT, AST), serum albumin, total proteins, serum bilirubin (total, direct), alkaline phosphatase (ALP) and fasting glucose. Biochemical tests were formed using Synchron CX5 auto-analyzer, Beckman Instrumentation, California, USA. Complete blood count (CBC) with stress on total leucocytic count was performed using Sysmex KX-21N, USA. Prothrombin time (PT) and partial thromboplastin time (PTT) were performed on the Sysmex CA 1500, Dade Behring, Germany. All patients were screened for $\mathrm{HCV} \mathrm{Ab}$ by ELISA, HBsAg, $\mathrm{HBcAb}$, Bilharsial Ab.

Fasting insulin level was determined using active Insulin Enzyme-Linked Immunosorbent Assay (ELISA) DSL-101600 Diagnostic Systems laboratories, Inc. Corporate. Normal values of fasting Insulin 6-35 $(\mu \mathrm{u} / \mathrm{ml})$. Insulin resistance was determined by the homeostasis model assessment (HOMA-IR) according to the formula:

$H O M A-I R=$ fasting glucose $(\mathrm{mmol} /$ liter $) \times$ fasting insulin (milliU / liter)

22.5 (glucose in $\mathrm{mmol} / \mathrm{l}$ ) or 403 (glucose in $\mathrm{mg} / \mathrm{ml}$ )

A HOMA score close to 1 indicates normal insulin sensitivity. Insulin resistance is associated with high HOMA scores.

Inflammatory cytokines: High sensitivity C-reactive protein was determined using ELISA Kit (Diagnostic Biochem. Candada Inc.). Serum tumor necrosis factor $\alpha$ (TNF- $\alpha$ ), Interleukin 6 (IL 6) were determined using a sandwich (ELISA) kit (Koma Biotech Inc., Korea).

Abdominal ultrasound was done with special stress on liver texture and size, splenomegaly, portal vein diameter and amount of ascites.

Statistical analysis Data was analyzed through statistical package of social science software program, version 18 (SPSS). Statistical Package for the Social Science; SPSS Inc., Chicago, Illinois, USA. Data were summarized using mean and SD for quantitative variables and frequency and percentage for qualitative variables. A comparison between groups was performed using the one-way analysis of variance test for quantitative variables with the post-hoc Tukey's test for pairwise comparisons and the $\chi 2$-test for qualitative variables. The Spearman correlation coefficient was calculated to test the association between quantitative variables. $\mathrm{P}$ values equal to or less than 0.05 were considered statistically significant.

\section{Results}

There was no significant difference in age and BMI between the 3 groups.

Table 1. Showing the demographic data of the patients.

\begin{tabular}{llll}
\hline Parameter & Group I (mean \pm SD) & Group II (mean \pm SD) & Group III (mean \pm SD) \\
\hline Age (years) & $47.700 \pm 12.1404$ & $44.867 \pm 13.763$ & $48.867 \pm 9.881$ \\
BMI & $26.792 \pm 4.974$ & $26.083 \pm 6.474$ & $25.719 \pm 9.036$ \\
\hline
\end{tabular}

Our results showed that CRP, TNF- $\alpha$, IL6, FBG, Fasting insulin, Insulin Resistance and Insulin Secretion were statistically significant different between the three groups with $\mathrm{p}$ value $<0.001$ except for the TNF- $\alpha$ its $\mathrm{p}$ value was $<0.05$.

Using the post-hoc Tukey's test for pairwise comparisons, CRP, TNF- $\alpha$, IL6, FBG, Fasting insulin, Insulin Resistance 
and Insulin Secretion were statistically significant higher in diabetic patients with $\mathrm{HCV}$ infection in comparison with non-diabetic patients with $\mathrm{HCV}$ infection and control group with $p$ value $<0.001$ except for the TNF- $\alpha$ in diabetic patients with $\mathrm{HCV}$ infection in comparison with non-diabetic patients with HCV infection, its $\mathrm{p}$ value was $<0.05$.

CRP and Insulin Resistance were statistically significant higher in non-diabetic patients with $\mathrm{HCV}$ infection in comparison with control group with $p$ value $<0.05$, also TNF- $\alpha, F B G$, and Insulin Secretion were statistically significant higher in non-diabetic patients with $\mathrm{HCV}$ infection in comparison with control group with $p$ value $<0.001$, however IL6 and Fasting insulin were higher in non-diabetic patients with $\mathrm{HCV}$ infection in comparison with control group with no statistical difference.

CRP, TNF- $\alpha$, and IL6 had statistically significant positive direct correlation with insulin resistance with $p$ value $<0.001$, $<0.001$ and $<0.004$ respectively.

Table 2. Showing Mean and Standard deviation of the main laboratory parameters among the three groups included in our study.

\begin{tabular}{llll}
\hline \multirow{2}{*}{ parameter } & Group $(\mathbf{I}) \mathbf{n}=\mathbf{5 0}$ & Group $($ II) $\mathbf{n}=\mathbf{5 0}$ & Group (III) $\mathbf{n}=\mathbf{2 5}$ \\
\cline { 2 - 4 } & Mean \pm S.D & Mean \pm S.D & Mean \pm S.D \\
\hline CRP $\mathrm{mg} / \mathrm{L}$ & $9.44 \pm 2.165 \mathrm{~A}$ & $7.70 \pm 2.726 \mathrm{~B}$ & $5.82 \pm 1.316 \mathrm{C}$ \\
TNF- $\alpha$ pg/ml) & $626.12 \pm 238.738 \mathrm{~A}$ & $618 \pm 302.805 \mathrm{~B}$ & $422.76 \pm 243.190 \mathrm{C}$ \\
IL6 pg/m & $215.63 \pm 62.004 \mathrm{~A}$ & $167.62 \pm 47.719 \mathrm{~B}$ & $173.72 \pm 57.427 \mathrm{~B}$ \\
FBG mg/dl & $168.3 \pm 52.234 \mathrm{~A}$ & $89.95 \pm 11.898 \mathrm{~B}$ & $<0.05$ \\
Fasting insulin $(\mu \mathrm{Lu} / \mathrm{ml})$ & $20.850 \pm 5.092 \mathrm{~A}$ & $11.357 \pm 2.302 \mathrm{~B}$ & $<0.001$ \\
Insulin Resistance & $3.020 \pm 0.719 \mathrm{~A}$ & $1.457 \pm 0.287 \mathrm{~B}$ & $6.846 \pm 1.088 \mathrm{~B}$ \\
Insulin Secretion & $36.552 \pm 18.855 \mathrm{~A}$ & $97.476 \pm 25.847 \mathrm{~B}$ & $1.064 \pm 0.160 \mathrm{C}$ \\
\hline
\end{tabular}

Values are expressed as mean $\pm \mathrm{SD}$; ANOVA, analysis of variance. The post-hoc Tukey's test: groups having different letters are statistically significantly different at a $\mathrm{P}$ value of $0.05 ; *$ The $\mathrm{P}$ value is statistically significant at $\mathrm{P}<0.05$ and highly significant with $\mathrm{P}<0.001$.

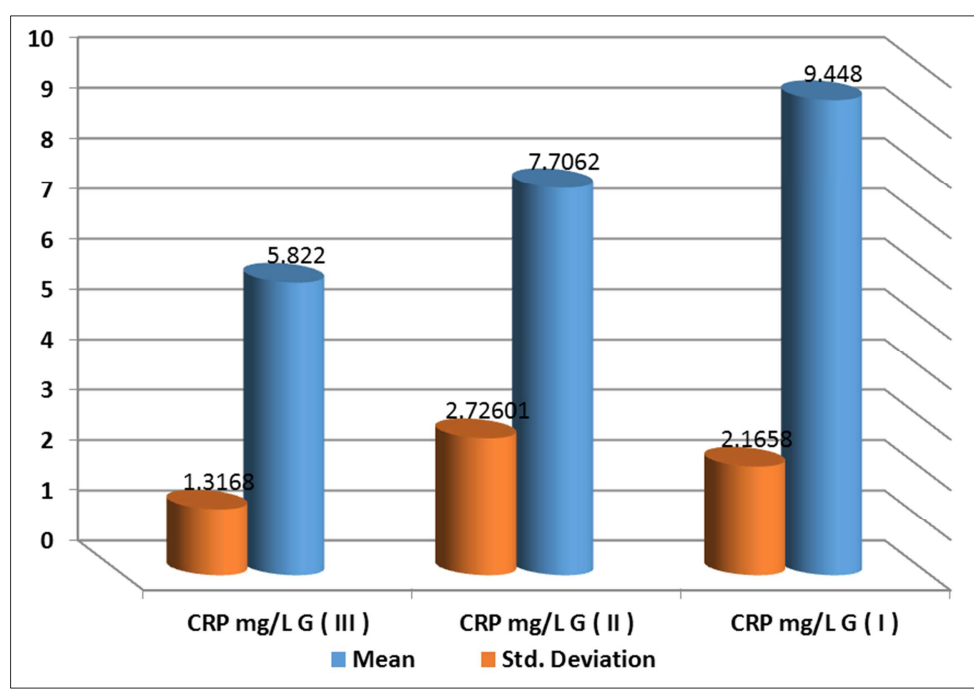

Figure 1. Represents CRP mean and S.D. level among the three groups.

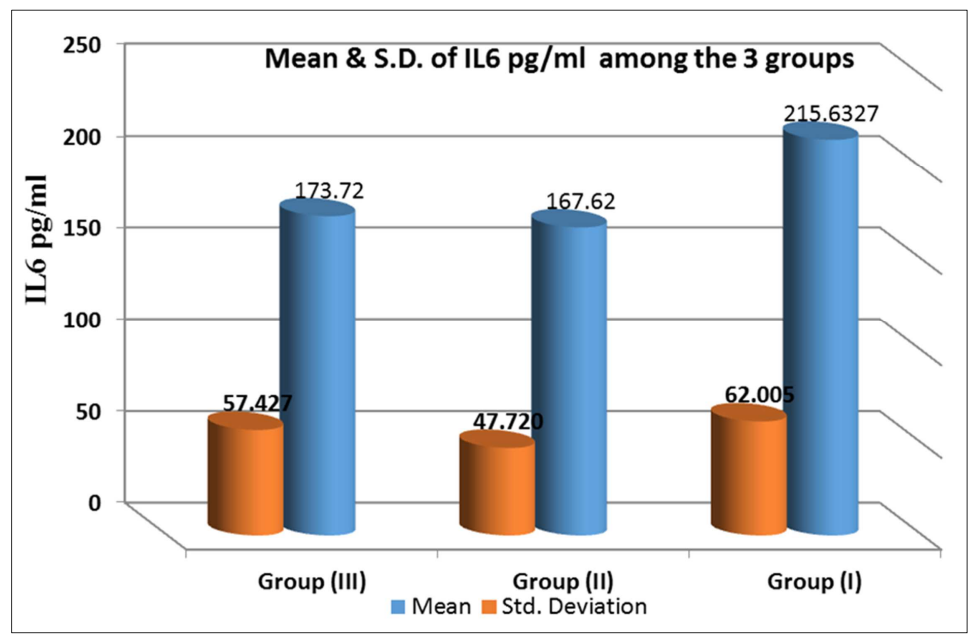

Figure 2. Represents mean and SD of interleukin 6 level among the 3 groups. 


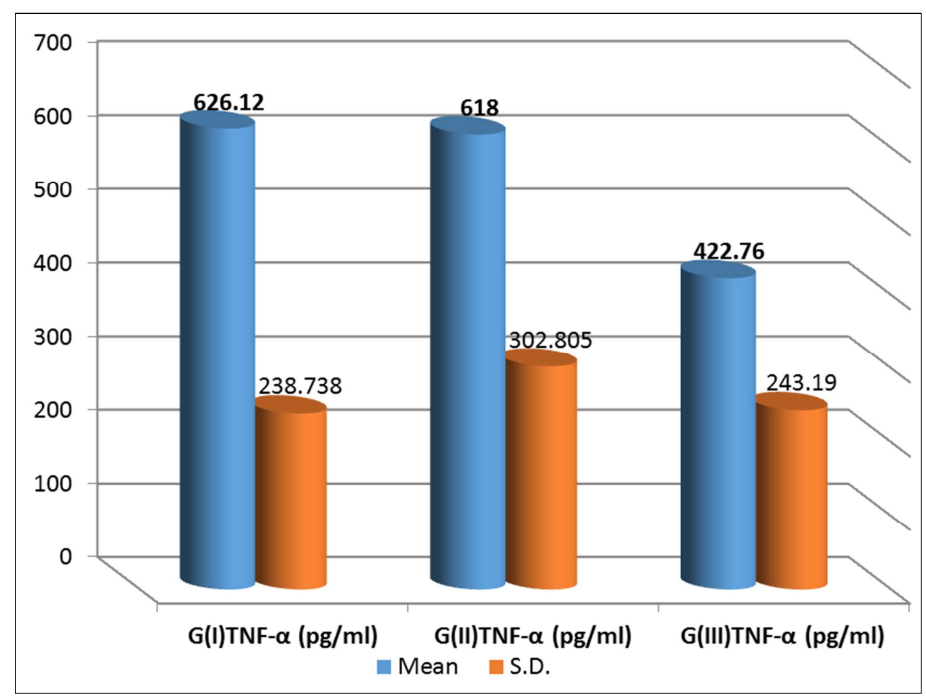

Figure 3. Representing mean and S.D. of the TNF- $\alpha(\mathrm{pg} / \mathrm{ml})$ in the three groups.

Table 3. Showing Pearson correlation coefficient between insulin resistance and CRP, TNF- $\alpha$-and IL-6.

\begin{tabular}{llll}
\hline Parameter & CRP & TNF- $\boldsymbol{\alpha}$ & IL-6 \\
\hline Insulin resistance & & & \\
$\mathrm{r}$ & 0.592 & 0.581 & 0.404 \\
$\mathrm{p}$-value & $<0.001$ & $<0.001$ & $<0.004$ \\
\hline
\end{tabular}

$\mathrm{r}$, Spearman correlation coefficient; *The $\mathrm{P}$ value is statistically significant.

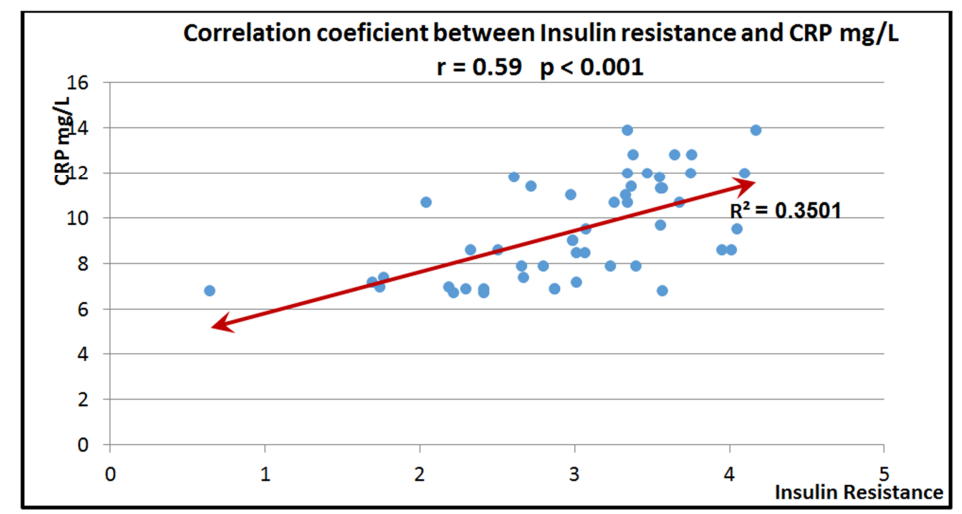

Figure 4. Showing correlation between Insulin resistance and CRP $m g / L$.

Represents statistically significant positive correlation between $\mathrm{C}$-reactive protein and insulin resistance.

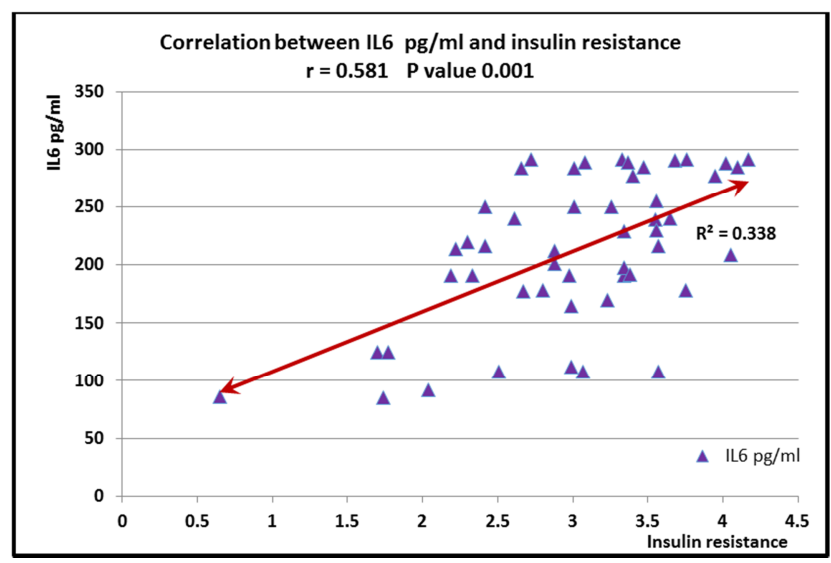

Figure 5. Showing Correlation between IL6 pg/ml and insulin resistance.
Represents statistically significant positive correlation coefficient between insulin resistance and interleukin $6(\mathrm{r}=$ 0.581 , P-value 0.001 ).

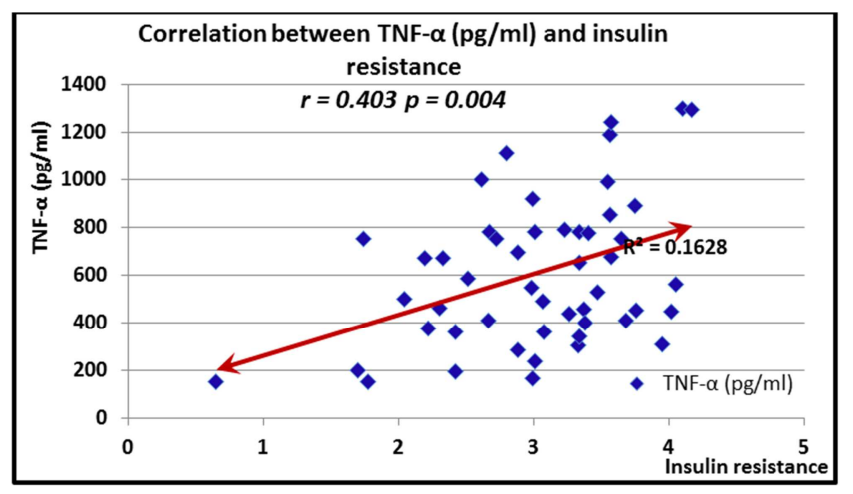

Figure 6. Showing correlation between $T N F-\alpha \quad(p g / m l)$ and insulin resistance. 
Represents statistically significant positive correlation coefficient between insulin resistance and TNF- $\alpha$ level $(\mathrm{r}=$ $0.403, \mathrm{P}$ - value of 0.004 ).

\section{Discussion}

Our study showed that diabetic patients with HCV infection (group I) have higher significant insulin resistance level than non-diabetic patients with $\mathrm{HCV}$ infection (group II) and diabetic patients without HCV infection (group III). Therefore the level of insulin resistance was highest among HCV diabetic patients. This finding was supported by Delgado et al., 2004 [5], who showed that $62 \%$ of patients with HCV had IR. Moreover a study performed at the tropical department at Ain Shams University Hospitals on Egyptian patients with $\mathrm{HCV}$ found that IR is present in $59 \%$ of the study population as compared to the control groups [6].

Insulin resistance mediated by proinflammatory cytokines, but not a deficit in insulin secretion, is the main pathogenic mechanism involved in the pathogenesis of diabetes associated with HCV infection [7].

This study compared the levels of the acute phase protein CRP and two of the pro inflammatory cytokines of the innate immunity, namely TNF- $\alpha$ and IL- 6 , in the three groups to establish a possible relationship between activation of the innate immune system and the development of insulin resistance in patients with $\mathrm{HCV}$ infection.

The ongoing inflammation was confirmed by the elevated levels of CRP. The level of high sensitivity CRP (hs-CRP) was significantly higher in HCV diabetic patients (group I) when compared to HCV patients without DM (group II) and diabetic patients without $\mathrm{HCV}$ (group III) with pvalue $<0.001$. CRP in group (II) was significantly higher when compared to group (III) with P-value $<0.05$. Also there was a statistically significant positive correlation between CRP level and insulin resistance $(r=0.592, \mathrm{P}$-value $<0.001)$ as shown in figure (4) which proves that CRP level increase among HCV patients shared in the development of insulin resistance in patients with chronic liver disease.

This finding is supported by Yudkin et al in 1999 [8] who demonstrated that in 107 non-diabetic subjects, CRP levels were related to insulin resistance. Concentrations of CRP correlated both with those of IL-6 $(p<0.0005)$ and of TNF$\alpha(\mathrm{P}<0.0001)$. These observations could suggest that the cytokines, arising in part from adipose tissue, might themselves be partly responsible for the metabolic, hemodynamic, and hemostatic abnormalities that cluster with insulin resistance.

The levels of IL6 in group (I) were significantly higher when compared to group (II) and (III) with P-value $<0.001$ while there were no statistical significance between levels of IL-6 in groups II and III. However there was a statistically significant positive correlation between IL6 level and insulin resistance $(\mathrm{r}=0.581, \mathrm{P}$-value $<0.001)$ as shown in figure (5). These findings are supported by Oyanagi et al in 1999 [9], who demonstrated that serum IL-6 levels were significantly elevated in chronic hepatitis $\mathrm{C}$ compared to those in normal controls. Kasprzak et al in 2004 [10] demonstrated an augmented expression of all pro-inflammatory cytokines and of IL- $1 \alpha$ and IL-6, particularly in the livers of chronic hepatitis $\mathrm{C}$ patients of either age group, as compared to their expressions in the control group. Fernandez-Real et al in 2001 [11], detected higher plasma IL-6 concentrations in patients with the insulin resistance syndrome and type 2 diabetes. His study concluded that insulin resistance was a significant and an independent predictor of peripheral IL-6 levels. Furthermore, Vozarova et al in 2001, [12] showed a positive correlation between plasma IL-6 concentrations and fasting insulin concentrations as an index of insulin resistance.

HCV may induce a Th1 lymphocyte immune-mediated response which leads to activation of the tumor necrosis factor (TNF)- $\alpha$ system and elevation of interleukin- 6 levels. A high TNF- $\alpha$ level was considered to be one of the bases of IR, "Meanwhile, HCV-induced inflammatory changes may subsequently lead to increased oxidative stress and increased peroxidation, which evoke systemic inflammatory responses (SIR) more often than other liver diseases [13]."

Levels of TNF- $\alpha$ were significantly higher in group I when compared to group II with (P-value $<0.05)$ and group III with (P-value $<0.001)$ as well as in group II. They were significantly higher when compared to group III with (Pvalue $<0.001)$. Furthermore, a statistically significant positive correlation was observed between TNF- $\alpha$ and insulin resistance ( $\mathrm{r}=0.403$, P-value 0.004$)$ as shown in figure (6).

These findings are supported by Lecube $A$ et al, 2006, [13], which provides the first clinical evidence of elevated levels of TNF- $\alpha$ and IL-6 in non-diabetic HCV positive patients. Dumoulin et al, 2001 and Knobler et al, 2003, [14], [15] have shown an increase in serum levels of TNF- $\alpha$ and its receptors in $\mathrm{HCV}$-infected patients

A study of liver biopsy specimens from non-diabetic $\mathrm{HCV}$ patients revealed significant impairments in the insulin signaling pathway, which are strikingly similar to the known effects of TNF- $\alpha$ and can lead to insulin resistance (16).

Festa et al in 2000, [17] showed that chronic subclinical inflammation is a part of the insulin resistance syndrome. Moreover, in cross-sectional studies, levels of inflammatory bio-markers such as C-reactive protein and the proinflammatory cytokines interleukin-6 (IL-6) and tumor necrosis factor $\alpha$ (TNF- $\alpha$ ), correlated with insulin sensitivity and with features of the insulin resistance syndrome [18].

This study is one of the few studies that measured serum levels of TNF- $\alpha$ in patients with chronic HCV either diabetics or non -diabetics. The previous studies support the idea that increased TNF- $\alpha$ levels might play a role in the insulin resistance of patients with chronic hepatitis induced by HCV. Our results thus confirm this by denoting the highly significant positive correlation between the level of CRP, IL$6, \mathrm{TNF}-\alpha$ and insulin resistance.

\section{Conclusion}

From this study, we can conclude that insulin resistance is 
more correlated to $\mathrm{HCV}$ diabetic patients than $\mathrm{HCV}$ nondiabetic patients and that there is a highly significant positive correlation between level of CRP, IL-6, TNF- $\alpha$ and insulin resistance. Investigating the association between $\mathrm{HCV}$ and $\mathrm{DM}$ is highly important. It represents a major public health problem that probably affects hundreds of thousands of patients. Many more $\mathrm{HCV}$-infected patients may have impaired glucose tolerance before becoming overtly diabetic. DM may also adversely affect the course of chronic hepatitis $\mathrm{C}$ and be associated with increased liver steatosis and fibrosis, and recent evidence suggests that steatosis and diabetes may also significantly enhance the risk of hepatocellular cancer. These patients may be less responsive to therapy and may show an increased prevalence of hepatocellular cancer. Finally, elucidating the risk factors and associated features of the HCV-diabetes association may shed a light on mechanisms of the disease. The HCV-DM association may constitute an example in which an exaggerated TNF response is deleterious to the host. Future research should focus on reaffirming this conclusion and defining effective interventions and strategies to block the local TNF response.

Therefore, IR should be assessed in patients infected with hepatits $\mathrm{C}$ virus, since IR is a risk factor for fibrosis progression. Antiviral treatment of the patients at risk is recommended as soon as possible, to selectively improve treatment outcome in these patients and thus prevent fibrosis progression while specific pharmaceutical treatment of insulin resistance is not yet established. Towards the future, $\mathrm{HCV}$ infection needs to be viewed not only as a liver disease but also as a metabolic disease.

\section{References}

[1] Sher Zaman Safi, Humaira Shah, Gracie OngSiok Yan, Rajes Qvist (2015): Insulin resistance provides the connection between hepatitis $\mathrm{C}$ virus and diabetes. Hepat Mon; 15 (1): e23941.

[2] Hui JM, Sud A, Farrell GC, Bandara P, et al (2003): Insulin resistance is associated with chronic hepatitis $C$ virus infection and fibrosis progression. Gastroenterology; 125: 1695-704.

[3] Gentilucci UV, Picardi A, and Pozzilli P (2006): Glucose abnormalities in patients with hepatitis $\mathrm{C}$ virus infection: epidemiology and pathogenesis: response to Lecube et al. Diabetes Care; 29 (11): 2558-2559.

[4] Simo R, Lecube A, Genesca, Esteban JR, and Hernandez C (2006): Sustained Virological Response Correlates With Reduction in the Incidence of Glucose Abnormalities in Patients with Chronic Hepatitis C Virus Infection. Diabetes Care; 29 (11): 2462-66.

[5] Delgado-Borrego A, Casson D, Schoenfeld D, Ma S, Adam T, Sergio JH et al (2004): Hepatitis C virus is independently associated with increased insulin resistance after liver transplantation. Transplantation; 77 (5): 703-10.
[6] Mahmoud HM (2005): Prevalence of insulin resistance in nondiabetic HCV patients. (Master's thesis). Ain Shams University.

[7] Lecube A, Cristina Hernández, Joan Genescà and Rafael Simó (2006): Glucose Abnormalities in Patients with Hepatitis C Virus Infection. DiabetesCare; 29 (5): 1140-9 (a).

[8] Yudkin JS, Stehouwer CD, Emeis JJ, Coppack SW (1999): Creactive protein in healthy subjects: associations with obesity, insulin resistance, and endothelial dysfunction: a potential role for cytokines originating from adipose tissue? Arterioscler Thromb Vasc Biol; 19: 972-8.

[9] Oyanagi Y, Takahashi T, Matsui S, Takahashi S, Boku S, Takahashi K et al (1999): Enhanced expression of interleukin6 in chronic hepatitis C. Liver.; 19 (6): 464-72.

[10] Kasprzak A, Seidel J, Spachacz R, Biczysko W, Makowska A, Kaczmarek $E$ et al. (2004): Intracellular expression of proinflammatory cytokines (IL-1, TNF- $\alpha$, and IL-6) in chronic hepatitis C. Roczniki Akademii Medycznej w Biaymstoku; 49: 207-9.

[11] Fernandez-Real JM, Vayreda M, Richart C, Gutierrez C, Broch M, Vendrell J et al (2001): Circulating Interleukin 6 Levels, Blood Pressure, and Insulin Sensitivity in Apparently Healthy Men and Women. The Journal of Clinical Endocrinology \& Metabolism; 86 (3): 1154-59.

[12] Vozarova B, Weyer C, Hanson K, Tataranni P, Bogardus $C$ and Pratley RE (2001): Circulating Interleukin-6 in relation to Adiposity, Insulin Action, and Insulin Secretion. Obes Res.; 9: 414-17.

[13] Lecube A, Hernández C, Genescà J and Simó R. (2006) Proinflammatory cytokines, insulin resistance, and insulin secretion in chronic hepatitis C patients: a case-control study. Diabetes Care; 29: 1096-101 (b).

[14] Dumoulin FL, Wennrich U, Nischalke HD Leifeld L, Fischer HP, Sauerbruch T et al (2001): Intrahepatic mRNA levels of interferon gamma and tumor necrosis factor alpha and response to antiviral treatment of chronic hepatitis C. J Hum Virol; 4: 195-99.

[15] Knobler H, Zhornicky T, Sandler A, Haran N, Ashur Y and Schattner $A$ (2003): TNF- $\alpha$ induced insulin resistance may mediate the $\mathrm{HCV}$-diabetes association. Am J Gastroenterol; 98: 2751-6.

[16] Aytug S, Reich D, Sapiro L E, Bernstein D and Begum $N$ (2003): Impaired IRS-1/PI3-kinase signaling in patients with HCV: a mechanism for increased prevalence of type 2 diabetes. Hepatology; 38: 1384-92.

[17] Festa A, D'Agostino R Jr, Howard G, Mykkänen L, Russell P. Tracy RP, Haffner SM (2000): Chronic subclinical inflammation as part of the insulin resistance syndrome: the Insulin Resistance Atherosclerosis Study (IRAS). Circulation; 102: $42-7$.

[18] Knobler H and Schattner A (2005): TNF- $\alpha$ chronic hepatitis C and diabetes: a novel triad QJM; 98 (1): 1-6. 